\title{
Anomalous Magnetic Field Effects on Photochemical Reactions in Ionic Liquid under Ultrahigh Fields of up to $28 \mathrm{~T}$
}

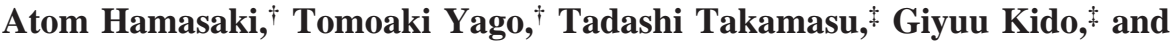 \\ Masanobu Wakasa*,† \\ Department of Chemistry, Graduate School of Science and Engineering, Saitama University, 255 Shimo-okubo, \\ Sakura-ku, Saitama-shi, Saitama 338-8570, Japan, and National Institute for Materials Science (NIMS), \\ 3-13 Sakura, Tsukuba, Ibaraki 305-0003, Japan
}

Received: October 16, 2007; In Final Form: December 21, 2007

\begin{abstract}
The magnetic field effects (MFEs) on photoinduced hydrogen abstraction reactions between benzophenone and thiophenol in an ionic liquid, $N, N, N$-trimethyl- $N$-propylammonium bis(trifluoromethanesulfonyl) imide (TMPA TFSI), were studied by a nanosecond laser flash photolysis technique under ultrahigh fields of up to 28 T. Extremely large and anomalous stepwise MFEs were observed for the first time. The escape yield of benzophenone ketyl radical decreased with increasing magnetic field strength $(B)$ at $0 \mathrm{~T}<B \leq 2 \mathrm{~T}$. The decrease was almost saturated at $2 \mathrm{~T}<B \leq 10 \mathrm{~T}$. At much higher fields $(10 \mathrm{~T}<B \leq 28 \mathrm{~T})$, the yield decreased again with increasing $B$, producing a $25 \%$ decrease at $28 \mathrm{~T}$.
\end{abstract}

\section{Introduction}

Magnetic field effects (MFEs) on photochemical reactions through radical pairs and biradicals have received considerable attention during the past three decades. Magnetic fields interact with the electron spins of radical pairs, and thus the spin conversion of the radical pairs between singlet (S) and triplet $\left(\mathrm{T}_{m}, m=0, \pm 1\right)$ states is influenced by the fields. The lifetime of the radical pairs and the yield of the escaped radicals consequently show appreciable MFEs. A large number of interesting phenomena on spin dynamics have already been reported. ${ }^{1,2}$ On the other hand, ionic liquids (ILs), which are considered as one of the most promising new solvents in green chemistry, have gained much attention because of their unusual chemical properties: nonvolatile, noncorrosive, nonflammable, stable in air and moisture, and designable. ${ }^{3-8}$ Some results observed in ILs are, however, quite different from those observed in conventional solvents. ${ }^{9-13}$ Hamaguchi et al. reported that the photoisomerization indeed proceeds in 1-butyl-3methylimidazolium hexafluorophosphate $\left([\mathrm{bmim}] \mathrm{PF}_{6}\right)$ with a rate much larger than that expected from its polarity and viscosity. ${ }^{9}$ McLean et al. reported the hydrogen abstraction reactions from ILs by benzophenone (BP) and the photoelectron transfer from ruthenium (II) tris(bipyridyl) to methylviologen in $[\mathrm{bmim}] \mathrm{PF}_{6} \cdot{ }^{10,11}$ García et al. reported that a series of photochemical reactions covering energy transfer, hydrogen transfer, and electron transfer was studied in $[\mathrm{bmim}] \mathrm{PF}_{6} \cdot{ }^{12}$ Recently, we found extremely large MFEs in ILs; the escape yield of benzophenone ketyl radical generated from the photoinduced hydrogen abstraction reaction of BP with thiophenol $(\mathrm{PhSH})$ in $N, N, N$-trimethyl- $N$-propylammonium bis(trifluoromethanesulfonyl) imide (TMPA TFSI) decreased by $20 \%$ at 1.7 T. ${ }^{13}$ The observed MFEs could not be explained by the high viscosity of the IL alone, but a local structure in the IL was

* To whom correspondence should be addressed. E-mail: mwakasa@ chem.saitama-u.ac.jp.

† Saitama University.

National Institute for Materials Science (NIMS). suggested as an origin of the large MFEs. To clarify the local structure of ILs and to find a new classified MFE, the complete MFEs data set from $0 \mathrm{~T}$ up to $28 \mathrm{~T}$ in ILs is necessary. Therefore, using a pulsed magnet, we studied the MFEs on the photoinduced hydrogen abstraction reaction of BP with $\mathrm{PhSH}$ in TMPA TFSI under ultrahigh magnetic fields of up to $28 \mathrm{~T}$.

\section{Experimental}

Materials. TMPA TFSI (Cica) was used as received. PhSH (Cica) was purified by vacuum distillation. BP (Cica) was recrystallized from methanol. The concentrations of $\mathrm{BP}$ and $\mathrm{PhSH}$ in the employed ILs were $2.0 \times 10^{-2}$ and $1.2 \times 10^{-1}$ mol $\mathrm{dm}^{-3}$, respectively. The viscosity and density of TMPA TFSI were measured by a Yamauchi VM-10A-L viscometer and an Anton Paar DMA 5000 density meter.

Nanosecond Laser Flash Photolysis. Laser flash photolysis experiments were carried out at $296 \mathrm{~K}$ using an apparatus described elsewhere. ${ }^{14,15}$ The third harmonic $(355 \mathrm{~nm})$ of a Quanta-Ray GCR-130-10 nanosecond Nd:YAG laser was used as an excitation light source. A double-beam probe system was used for measuring the transient absorption accurately. The transient absorption was recorded by a Hewlett-Packard HP54522A digitizing oscilloscope (2 GHz) with a Hamamatsu R636 photomultiplier. The argon-bubbled solution in a quartz cell was placed at the center of a pulsed magnet. The pulsed magnet had a room-temperature bore with a diameter of $20 \mathrm{~mm}$ and a length of $160 \mathrm{~mm}$. Pulsed magnetic fields were generated by supplying intense pulsed currents from a $10 \mathrm{mF}$ capacitor bank of $125 \mathrm{~kJ}$ at $5 \mathrm{kV}$. The maximum field was $32.2 \mathrm{~T}$ at 3.5 $\mathrm{kV}$. Applied magnetic field was measured with a search coil placed right next to the quartz cell.

\section{Results and Discussion}

The reaction scheme of photoinduced hydrogen abstraction reaction between $\mathrm{BP}$ and $\mathrm{PhSH}$ can be represented as follows: 


$$
\begin{gathered}
\mathrm{BP}+\mathrm{h} v(355 \mathrm{~nm}) \rightarrow{ }^{1} \mathrm{BP}^{*} \rightarrow{ }^{3} \mathrm{BP}^{*} \\
{ }^{3} \mathrm{BP} *+\mathrm{PhSH} \rightarrow{ }^{3}(\mathrm{BPH} \bullet \bullet \mathrm{SPh}) \\
{ }^{3}(\mathrm{BPH} \bullet \bullet \mathrm{SPh}) \stackrel{{ }^{B}}{\rightarrow}(\mathrm{BPH} \bullet \bullet \mathrm{SPh}) \\
{ }^{3}(\mathrm{BPH} \bullet \bullet \mathrm{SPh}) \rightarrow \mathrm{BPH} \bullet+\bullet \mathrm{SPh}(\text { escaped radicals }) \\
{ }^{1}(\mathrm{BPH} \bullet \bullet \mathrm{SPh}) \rightarrow \text { cage products }
\end{gathered}
$$

Here, ${ }^{1} \mathrm{BP} *,{ }^{3} \mathrm{BP} *, \mathrm{BPH} \bullet$, and $\bullet \mathrm{SPh}$ represent the singlet and triplet excited states of benzophenone, benzophenone ketyl, and phenylthiyl radicals, respectively. ${ }^{1}(\mathrm{BPH} \bullet \bullet \mathrm{SPh})$ and ${ }^{3}(\mathrm{BPH} \bullet$ - $\mathrm{SPh}$ ) denote singlet and triplet radical pairs composed of benzophenone ketyl and phenylthiyl radicals, respectively. This reaction system showed appreciable MFEs, even in nonviscous homogeneous solutions such as methanol $(\eta=0.55 \mathrm{cP})$ and ethanol $(\eta=1.08 \mathrm{cP}) .{ }^{16}$ Thus the rate constant of the recombination can safely be estimated to be as large as $10^{9}-$ $10^{10} \mathrm{~s}^{-1}$ because no MFE should be observed when the recombination reaction (eq 5) is much slower than the escaping process $\left(>10^{9} \mathrm{~s}^{-1}\right)$ (eq 4$)$.

Since BPH• has transient absorption bands around 380 and $550 \mathrm{~nm},{ }^{17}$ the magnetic field dependence of the time profile of the transient absorption, $A(t)$, was measured at $380 \mathrm{~nm}$. At $0 \mathrm{~T}$ $<B \leq 0.05 \mathrm{~T}$, no change of the $A(t)$ curve was observed within the experimental error, but at above $0.1 \mathrm{~T}$, it showed clear MFEs. Figure 1 typically shows the $A(t)$ curves observed at 0 and $28 \mathrm{~T}$. The decay of each $A(t)$ curve has fast and slow decay components. The fast component is ascribable to the decay of radical pairs, and the slow one can be ascribed to the escaped $\mathrm{BPH} \bullet$. As shown in Figure 1, the first decay component due to the radical pairs is clearly affected by the fields. Since the lifetime of triplet-triplet absorption ( $\left.{ }^{3} \mathrm{BP} *\right)$ observed at $520 \mathrm{~nm}$ was $85 \mathrm{~ns}$, the $A(0.75 \mu \mathrm{s})$ value is proportional to the escaped radical yield $(Y(B))$. Thus, the ratio $R(B)=Y(B) / Y(0 \mathrm{~T})=A(0.75$ $\mu \mathrm{s}, B \mathrm{~T}) / A(0.75 \mu \mathrm{s}, 0 \mathrm{~T})$ gives the MFE on the yield of the escaped $\mathrm{BPH} \bullet$. In the present study, $R(28 \mathrm{~T})$ was obtained to be $0.75 \pm 0.02$. This means that the yield of the escaped BPH• decreased by $25 \%$ at $28 \mathrm{~T}$ compared with that at $0 \mathrm{~T}$.

Next, the $R(B)$ values are plotted against $B$ in Figure 2. The stepwise field dependence of $R(B)$ can be summarized as follows: (1) $R(B)$ decreased with increasing $B$ at $0 \mathrm{~T}<B \leq 2$ T. (2) The decrease was almost saturated at $2 \mathrm{~T}<B \leq 10 \mathrm{~T}$, producing $R(10 \mathrm{~T})$ of 0.79 . (3) At much higher fields $(10 \mathrm{~T}<$ $B \leq 28 \mathrm{~T}), R(B)$ decreased again with increasing $B$. In Figure 3 , the reaction scheme, triplet-singlet $(\mathrm{T}-\mathrm{S})$ spin conversion, and spin relaxation of the present radical pairs at (a) $B=0 \mathrm{~T}$, (b) $0 \mathrm{~T}<B \leq 2 \mathrm{~T}$, (c) $2 \mathrm{~T}<B \leq 10 \mathrm{~T}$, and (d) $10 \mathrm{~T}<B \leq$ $28 \mathrm{~T}$ are schematically shown. Here $\mathrm{S}$ and $\mathrm{T}$ denote singlet and triplet radical pairs, respectively. $Y$ represents the yield of the process described in the figure. $k_{\mathrm{esc}}, k_{\mathrm{HFCM}}, k_{\triangle \mathrm{gM}}$, and $k_{\mathrm{SR}}$ are the rate constants of the escaping process, $\mathrm{T}-\mathrm{S}$ spin conversion due to the hyperfine coupling mechanism (HFCM) and the $\Delta g$ mechanism $(\Delta g \mathrm{M})$, and spin relaxation, respectively.

The decrease of $R(B)$ at $0 \mathrm{~T}<B \leq 2 \mathrm{~T}$ and the saturation of MFEs at $2 \mathrm{~T}<B \leq 10 \mathrm{~T}$ can safely be explained by the $\Delta g \mathrm{M}$, which originates from the difference between the isotropic $g$ factors of two radicals in a pair, ${ }^{1,2,16,18}$ because the present radical pair of BPH• $(g=2.0030)$ and $\bullet S P h(g=2.0082)$ has a large $\Delta g$ value of $0.0052 .{ }^{18}$ Moreover, the plots of $R(B)$ vs $B^{1 / 2}$ have a good linear relationship, as shown in Figure 2 (inset). Such field dependence of the linear relationship is well-known to the MFEs due to the $\Delta g \mathrm{M} .^{16}$

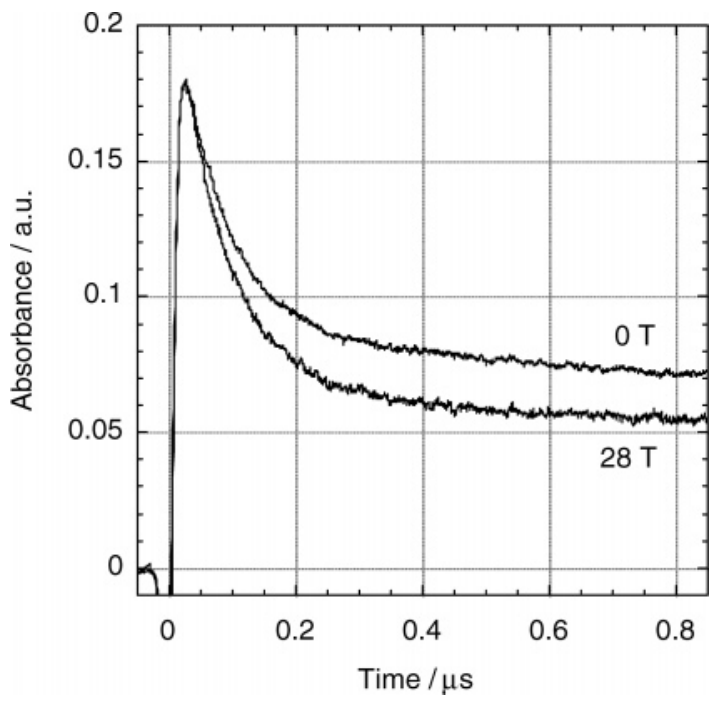

Figure 1. $A(t)$ curves observed at $380 \mathrm{~nm}$ for the photoinduced hydrogen abstraction reaction of BP with PhSH in TMPA TFSI at 0 and $28 \mathrm{~T}$.

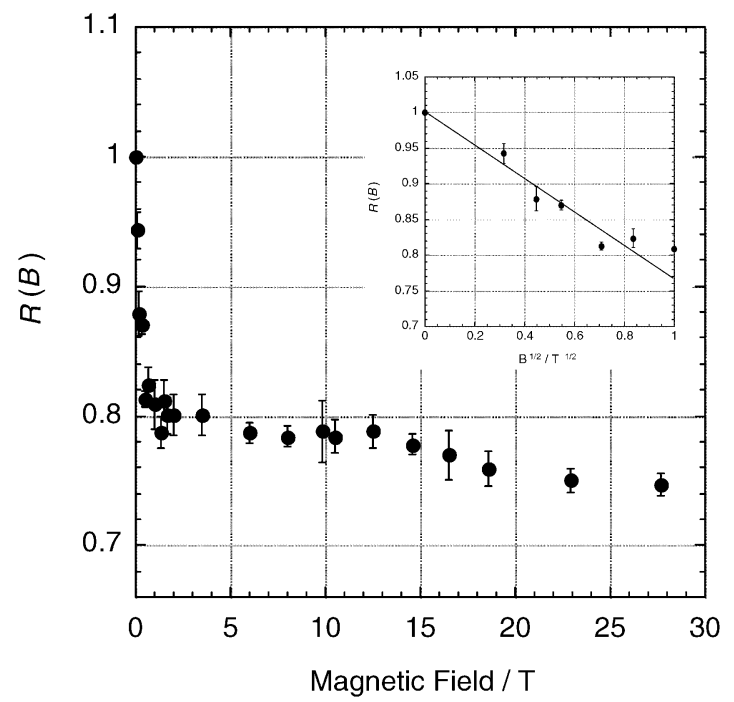

Figure 2. Magnetic field dependence of the yield of escaped BPH• $(R(B)=A(0.75 \mu \mathrm{s}, B \mathrm{~T}) / A(0.75 \mu \mathrm{s}, 0 \mathrm{~T}))$ observed at $380 \mathrm{~nm}$ in TMPA TFSI. Inset: Plots of $R(B)$ against $B^{1 / 2}$ at $0 \mathrm{~T} \leq B \leq 1 \mathrm{~T}$.

The saturation of MFEs due to the $\triangle g \mathrm{M}$ has previously been reported for the similar reaction of 4-methoxybenzophenone with $\mathrm{PhSH}$ in nonviscous conventional solvents such as 2-methyl1-propanol. ${ }^{18}$ In 2-methyl-1-propanol $(\eta<3.3 \mathrm{cP})$, the escaping process from the radical pairs is much faster than the $\mathrm{T}-\mathrm{S}$ spin conversion process. At $0 \mathrm{~T}$, almost all of triplet radical pairs are escaped from the pairs. In the high field region, the spin conversion of $\mathrm{T}_{0}-\mathrm{S}$ is accelerated by the $\Delta g \mathrm{M}$, and it becomes much faster than the escaping process. As the limiting value of the yield of the $\mathrm{T}_{0}-\mathrm{S}$ spin conversion process, $33 \%$ of the triplet radical pairs $\left(\mathrm{T}_{0}\right)$ can convert to the singlet pairs and disappear by the recombination process. Therefore, the saturation of $R(B)$ was observed in the high field region, producing an $R(B)$ of 0.66. In the present study, however, $R(B)$ observed at the saturated field was as large as 0.79 . Such large $R(B)$ values can be explained by the escaping rate of the triplet pairs in TMPA TFSI. Since the viscosity of TMPA TFSI $(72.6 \mathrm{cP})$ is much larger than that of 2-methyl-1-propanol $(3.3 \mathrm{cP})$, the escaping process of the triplet pairs in TMPA TFSI should be slow. Thus a part of the triplet pairs can convert to the singlet pairs, even at $0 \mathrm{~T}$, and disappear by the recombination process, as shown 
(a) $\mathrm{B}=0 \mathrm{~T}$

$$
R(B)=\frac{Y_{\text {esc }}(B)}{Y_{\text {esc }}(0 T)}
$$

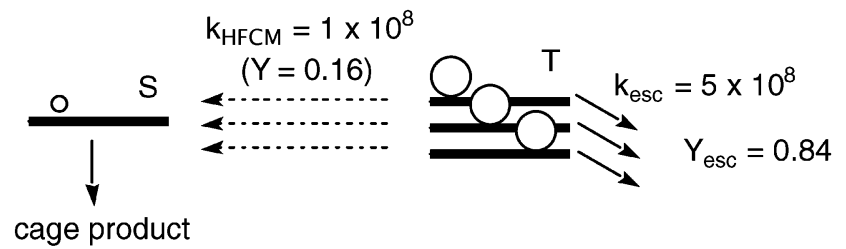

(b) $0 \mathrm{~T}<\mathrm{B} \leq 2 \mathrm{~T}$

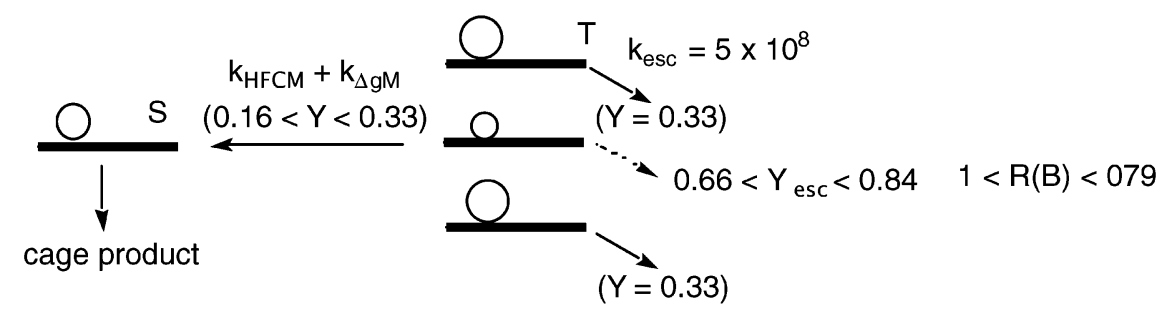

(c) $2 \mathrm{~T}<\mathrm{B} \leq 10 \mathrm{~T}$

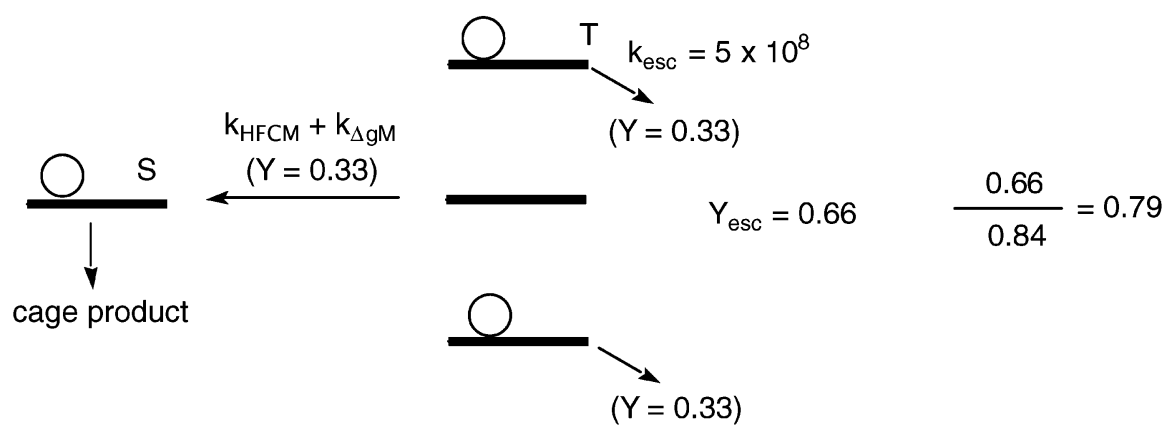

(d) $10 \mathrm{~T}<\mathrm{B} \leq 28 \mathrm{~T}$

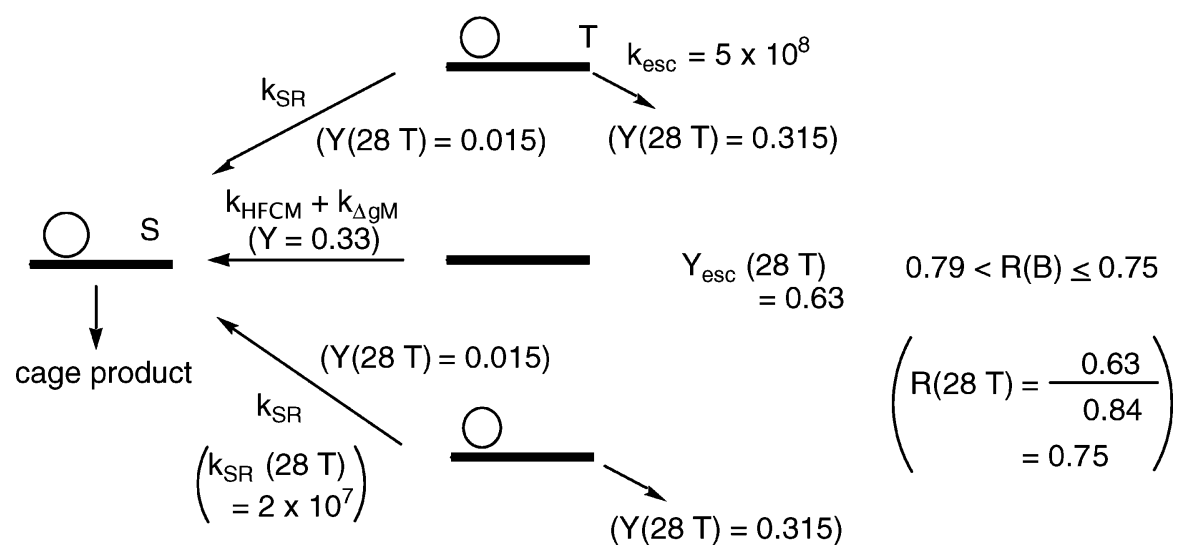

Figure 3. Reaction scheme, $\mathrm{T}-\mathrm{S}$ spin conversion and spin relaxation of the present radical pairs generated from a triplet precursor at (a) $B=0$ T, (b) $0 \mathrm{~T}<B \leq 2 \mathrm{~T}$, (c) $2 \mathrm{~T}<B \leq 10 \mathrm{~T}$, and (d) $10 \mathrm{~T}<B \leq 28 \mathrm{~T}$.

in Figure 3(a). The escaped radical $\left(Y_{\text {esc }}\right)$ at $0 \mathrm{~T}$ in TMPA TFSI was estimated to be 0.84 , which was discussed later. With increasing $B$ at $0 \mathrm{~T}<B \leq 2 \mathrm{~T}$, as shown in Figure 3(b), the spin conversion of $\mathrm{T}_{0}-\mathrm{S}$ is accelerated by the $\Delta g \mathrm{M}$, thus $Y_{\mathrm{esc}}$ decreases from 0.84. At the saturated fields of $2 \mathrm{~T}<B \leq 10$ $\mathrm{T}$, the rate constant of $\mathrm{T}_{0}-\mathrm{S}$ spin conversion due to the $\Delta g \mathrm{M}$ $\left(k_{\Delta \mathrm{gM}}\right)$ is much larger than the rate constant of the escaping process: $k_{\Delta \mathrm{gM}}$ at $10 \mathrm{~T}$ was calculated to be $2.3 \times 10^{9} \mathrm{~s}^{-1}$ from the $\Delta g$ value of 0.0052 . Thus almost all of triplet radical pairs in the $\mathrm{T}_{0}$ state $(33 \%)$ can convert to singlet pairs and disappear by the recombination process. As clearly seen in Figure 3(c), $Y_{\mathrm{esc}}$ became 0.66 , resulting in the $R(B)\left(=Y_{\mathrm{esc}}(B) / Y_{\mathrm{esc}}(0 \mathrm{~T})\right)$ of
0.79. Using these values, we can estimate that $16 \%$ of the triplet radical pairs at $0 \mathrm{~T}$ convert to singlet radical pairs and disappear by the recombination process. This means that $Y_{\text {esc }}$ is 0.84 . Since $k_{\mathrm{HFCM}}$ can roughly be estimated to be $1 \times 10^{8} \mathrm{~s}^{-1}$ from the HFC constants of BPH• and PhS $\bullet, k_{\text {esc }}$ is calculated to be $5 \times$ $10^{8} \mathrm{~s}^{-1}$ using the simple kinetic model as shown in Figure 3(a).

In a previous paper, ${ }^{18}$ the $R(B)$ values in 2-methyl-1-propanol were saturated above $20 \mathrm{~T}$, and the observed $R(B)$ values could be reproduced fairly well by the diffusion model. According to the diffusion model developed for the MFE in such conventional solvents, the saturated fields due to the $\Delta g \mathrm{M}$ should decrease with increasing the solvent viscosity. ${ }^{19,20}$ Thus we tried to 
estimate the viscosity needed for the saturation of the MFEs at $2 \mathrm{~T}$ using the diffusion model together with the $\Delta g \mathrm{M}$. However, the estimated viscosity of $400 \mathrm{cP}$ is much larger than that of TMPA TFSI $(72.6 \mathrm{cP})$. Therefore the saturation of the MFEs at $2 \mathrm{~T}$ cannot be explained by the viscosity of the IL alone. The cage effect of ILs is the most likely candidate to explain the observed saturation. If ILs have the local structure like a micellar solution, it can work as a cage for the photochemical reactions and cause the saturation of the MFEs at the low field region. In fact, the local structure or some domains in ILs were recently reported by Hamaguchi et al., ${ }^{21}$ Wang et al., ${ }^{22}$ Nishikawa et al., ${ }^{23}$ and Wishart et al. ${ }^{24}$

The secondary decrease of $R(B)$ at $10 \mathrm{~T}<B \leq 28 \mathrm{~T}$ may be due to the relaxation mechanism (RM), which originates from the spin relaxation of $T_{ \pm 1}-T_{0}$ and $T_{ \pm 1}-S$. According to the RM by Hayashi and Nagakura, ${ }^{25}$ the spin relaxation rates of $k_{\mathrm{R}}$ and $k_{\mathrm{R}^{\prime}}$ for a radical pair consisting of radical $\mathrm{A}$ and radical $\mathrm{B}$ can be represented as follows:1,2,25

$$
\begin{gathered}
k_{\mathrm{R}}=k_{\mathrm{dd}}+k_{\mathrm{A}}+k_{\mathrm{B}} \\
k_{\mathrm{R}^{\prime}}=k_{\mathrm{A}}+k_{\mathrm{B}}
\end{gathered}
$$

Here, $k_{\mathrm{R}}$ is the spin relaxation rate between $\mathrm{T}_{ \pm 1}$ and $\mathrm{T}_{0}$, and $k_{\mathrm{R}^{\prime}}$ is that between $\mathrm{T}_{ \pm 1}$ and $\mathrm{S}$. $k_{\mathrm{dd}}$ is the rate constant for the inter-radical relaxation induced by the electron spin-spin interaction. $k_{j}(j=\mathrm{A}, \mathrm{B})$ is the rate constant for the intra-radical relaxation of radical $j . k_{j}$ can be given by

$$
k_{j}=k_{j}^{\delta \mathrm{HFC}}+k_{j}^{\delta g}
$$

Here, $k_{j}^{\delta \mathrm{HFC}}$ and $k_{j}^{\delta g}$ are the rate constants of spin-lattice relaxation by the anisotropic hyperfine coupling and the anisotropic Zeeman interaction, respectively. The magnetic field dependence of $k_{\mathrm{R}}$ and $k_{\mathrm{R}^{\prime}}$ can be calculated from the analytical forms of $k_{\mathrm{dd}}, k_{j}^{\delta \mathrm{HFC}}$, and $k_{j}^{\delta g}$ as follows:

$$
\begin{gathered}
k_{\mathrm{dd}}=\frac{\mu_{\mathrm{B}}^{4} g_{\mathrm{A}}{ }^{2} g_{\mathrm{B}}{ }^{2}}{10 \hbar^{2} R^{6}} \frac{3 \tau_{\mathrm{AB}}}{1+\omega^{2} \tau_{\mathrm{AB}}{ }^{2}} \\
k_{j}^{\delta \mathrm{HFC}}=\frac{1}{30 \hbar^{2}} \delta a_{j}^{2} \frac{2 \tau_{j}}{1+\omega^{2} \tau_{j}^{2}} \\
k_{j}^{\delta g}=\frac{1}{30 \hbar^{2}} \mu_{\mathrm{B}}{ }^{2} B^{2} \delta g_{j}^{2} \frac{2 \tau_{j}}{1+\omega^{2} \tau_{j}^{2}} \\
\omega=\hbar^{-1} g \mu_{\mathrm{B}} B
\end{gathered}
$$

Here $g_{\mathrm{A}}$ and $g_{\mathrm{B}}$ represent the isopropic $g$ values of radicals A and $\mathrm{B}$, respectively. $\tau_{\mathrm{AB}}$ is the correlation time of the radical pair. $\delta a$ and $\delta g$ denote the difference of the anisotropic hyperfine coupling constants $\left(\left|A_{\|}-A_{\perp}\right|\right)$ and the difference of the anisotropic $g$ values $\left(\left|g_{\|}-g_{\perp}\right|\right)$, respectively. $\tau_{j}$ is the correlation time of each component radical. We can see from eqs 9-12 that $k_{\mathrm{dd}}$ and $k_{j}^{\mathrm{dHFC}}$ decrease with increasing $B$, but $k_{j}^{\delta g}$ increases with increasing $B$. The $k_{\mathrm{R}}+k_{\mathrm{R}^{\prime}}$ values were calculated with the following parameters (subscripts denote the individual radicals; A for $\mathrm{BPH} \bullet$ and $\mathrm{B}$ for $\mathrm{PhS} \bullet$ ): $g_{\mathrm{A}}=2.0030,{ }^{18} g_{\mathrm{B}}=$ $2.0082,{ }^{18} \delta a_{\mathrm{A}} / g \mu_{\mathrm{B}}=0.002 \mathrm{~T},{ }^{26,27} \delta a_{\mathrm{B}} / g \mu_{\mathrm{B}}=0.002 \mathrm{~T},{ }^{15,28} \delta g_{\mathrm{A}}$ $=0.002,{ }^{26,27} \delta g_{\mathrm{B}}=0.02,{ }^{15,28} \tau_{\mathrm{A}}=\tau_{\mathrm{B}}=1.0 \times 10^{-12} \mathrm{~s},{ }^{15,26,27}$ $\tau_{\mathrm{AB}}=1.0 \times 10^{-10} \mathrm{~s},{ }^{15,28}$ and $R=1.0 \mathrm{~nm} \cdot{ }^{15,27}$ Here the correlation time $\left(\tau_{j}\right)$ of $1 \times 10^{-12} \mathrm{~s}$ is much smaller than that estimated from the viscosity of TMPA TFSI $(\eta=72.6 \mathrm{cP})$.

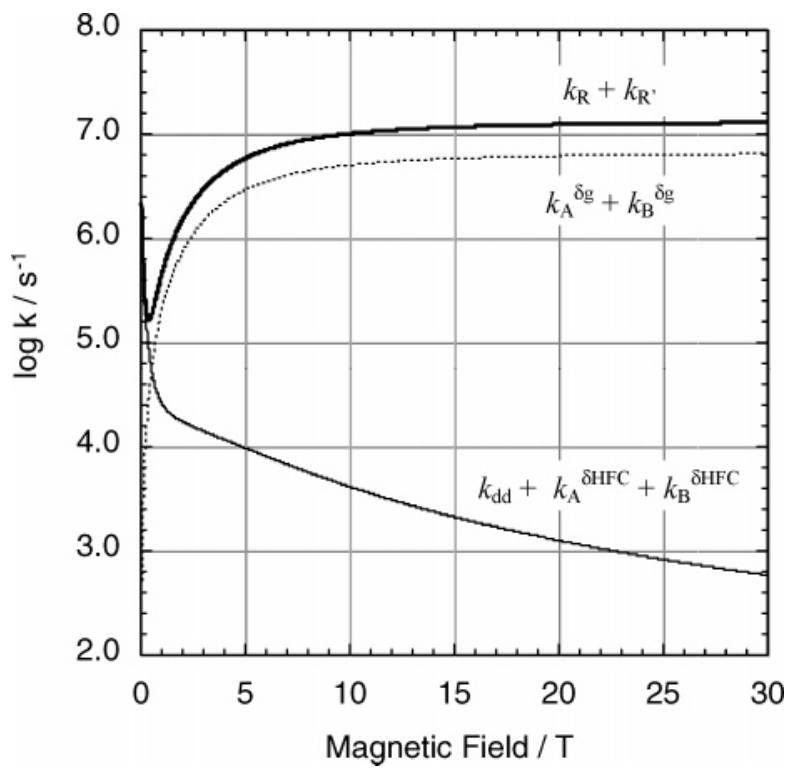

Figure 4. Calculated magnetic field dependence of the spin relaxation rates of $k_{\mathrm{R}}+k_{\mathrm{R}^{\prime}}, k_{\mathrm{dd}}+k_{\mathrm{A}}^{\delta \mathrm{HFC}}+k_{\mathrm{B}}^{\delta \mathrm{HFC}}$, and $k_{\mathrm{A}}^{\delta g}+k_{\mathrm{B}}^{\delta g}$ for a model radical pair having the following parameters (subscripts denote the individual radicals; radical A for BPH $\bullet$ and radical B for PhS $\bullet$ ): $g_{\mathrm{A}}=$ $2.0030, g_{\mathrm{B}}=2.0082, \delta a_{\mathrm{A}} / g \mu_{\mathrm{B}}=0.002 \mathrm{~T}, \delta a_{\mathrm{B}} / g \mu_{\mathrm{B}}=0.002 \mathrm{~T}, \delta g_{\mathrm{A}}=$ $0.002, \delta g_{\mathrm{B}}=0.02, \tau_{\mathrm{A}}=\tau_{\mathrm{B}}=1.0 \times 10^{-12} \mathrm{~s}, \tau_{\mathrm{AB}}=1.0 \times 10^{-10} \mathrm{~s}$, and $R=1.0 \mathrm{~nm}$.

Since local structure or some domains in the ILs were suggested, the microscopic viscosity in such structure or domains may be small, resulting in a small correlation time. In the previous paper, no MFE was observed in a viscous homogeneous solvent (cyclohexanol/2-methyl-1-propanol (100:1(v/v)), $\eta=54.8 \mathrm{cP}$ ) using the same reaction system. ${ }^{13}$ If the microscopic viscosity in TMPA TFSI is similar to the bulk viscosity $(72.6 \mathrm{cP})$, the MFEs should not be observed. Thus the microscopic viscosity in the IL is considered to be very small. Similar results were reported by Hamaguchi et al. ${ }^{9}$ In their paper, photoisomerization of trans-stilbene in an IL occurred efficiently in spite of its large viscosity. This may be due to the small microscopic viscosity of the IL. In Figure $4, \log \left(k_{\mathrm{R}}+k_{\mathrm{R}}\right), \log \left(k_{\mathrm{dd}}+k_{\mathrm{A}}^{\delta \mathrm{HFC}}+k_{\mathrm{B}}^{\delta \mathrm{HFC}}\right)$, and $\log \left(k_{\mathrm{A}}^{\delta g}+k_{\mathrm{B}}^{\delta g}\right)$ values are plotted against $B$. The calculated $k_{\mathrm{R}}+k_{\mathrm{R}^{\prime}}$ values show clear $B$ dependence as follows: (1) At the low field region, $k_{\mathrm{R}}+k_{\mathrm{R}^{\prime}}$ decreases with increasing $B$. (2) At the high field region, $k_{\mathrm{R}}+k_{\mathrm{R}^{\prime}}$ increases gradually with increasing $B$. (3) At $28 \mathrm{~T}, k_{\mathrm{R}}+k_{\mathrm{R}^{\prime}}$ is estimated to be $1.2 \times 10^{7}$ $\mathrm{s}^{-1}$. As seen in Figure 4, the spin relaxation of $\mathrm{T}_{ \pm 1}-\mathrm{T}_{0}$ and $\mathrm{T}_{ \pm 1}-\mathrm{S}$ can compete with the escaping process only at the high field region. The spin relaxation causes the decrease of $Y_{\text {esc }}$. The MFEs observed at $10 \mathrm{~T}<B \leq 28 \mathrm{~T}$ can be explained by this mechanism (RM) and rationalized by Figure 3(d). Using the simple kinetic model as shown in Figure 3(d) and the observed $R(28 \mathrm{~T})$ value of $0.75, k_{\mathrm{SL}}$ at $28 \mathrm{~T}$ is estimated to be $2 \times 10^{7} \mathrm{~s}^{-1}$. This $k_{\mathrm{SL}}$ value agrees well with the calculated $k_{\mathrm{R}}$ $+k_{\mathrm{R}^{\prime}}$ value of $1.2 \times 10^{7} \mathrm{~s}^{-1}$. Therefore, the MFEs observed in the present study at $10 \mathrm{~T}<B \leq 28 \mathrm{~T}$ can be explained by the RM with the small correlation time.

The alternative mechanism for the observed sequential increase in the MFEs is an anisotropic $\Delta g$-induced $\mathrm{S}-\mathrm{T}_{0}$ intersystem crossing transitions in rotation-hindered radical pairs. If rotation is slow enough, different pairs will experience different local $\Delta g$. The rotation-hindered radical pairs with small $\Delta g$ might have insufficiently rapid intersystem crossing in a given field but it can be accelerated at higher fields. Since such local $\Delta g$ values could not be obtained directly from the 
experiments, more theoretical studies using the stochastic Liouville equation are now in progress, but these are beyond the scope of the present experimental paper.

\section{Conclusions}

MFEs on the photoinduced hydrogen abstract reactions of $\mathrm{BP}$ with $\mathrm{PhSH}$ in TMPA TFSI were investigated under ultrahigh fields of up to $28 \mathrm{~T}$. Large and anomalous stepwise MFEs were observed. The MFEs observed at $0 \mathrm{~T}<B \leq 2 \mathrm{~T}$ and the saturation of MFEs at $2 \mathrm{~T}<B \leq 10 \mathrm{~T}$ can be explained by the $\Delta g \mathrm{M}$, and those observed at $10 \mathrm{~T}<B \leq 28 \mathrm{~T}$ can be explained by the RM. Using the simple kinetic model and the observed $R$ $(28 \mathrm{~T})$ value, the spin relaxation rate $\left(k_{\mathrm{SL}}\right)$ at $28 \mathrm{~T}$ is experimentally estimated to be $2 \times 10^{7} \mathrm{~s}^{-1}$ and is rationalized by the theoretically calculated $k_{\mathrm{R}}+k_{\mathrm{R}^{\prime}}$ value of $1.2 \times 10^{7} \mathrm{~s}^{-1}$. The MFEs due to the RM cannot be observed in conventional solvents, but can in micellar solutions. Therefore, it is noteworthy that the large cage effect of the IL plays an important role in the secondary decrease of $R(B)$.

Acknowledgment. This work was partially supported by a Grant-in-Aid for Scientific Research (No. 17073002) in Priority Area "Science of Ionic Liquids" (Area Number 452) from the Ministry of Education, Culture, Sports, Science, and Technology of Japan.

\section{References and Notes}

(1) Steiner, U. E.; Ulrich, T. Chem. Rev. 1989, 89, 51.

(2) Nagakura, S; Hayashi, H.; Azumi, T. Dynamic Spin Chemistry; Kodansha-Wiley: Tokyo, New York, 1998.

(3) Seddon, K. R. J. Chem. Technol. Biotechnol. 1997, 68, 351.

(4) Welton, T. Chem. Rev. 1999, 99, 2071.
(5) Wasserscheid, P.; Kein, W. Angew. Chem., Int. Ed. 2000, 39, 3772.

(6) Sheldon, R. Chem. Commun. 2001, 2399. 3667.

(7) Dupont, J.; Souza, R. F.; Saurez, P. A. Z. Chem. Rev. 2002, 102,

(8) Ohno, H. Electrochemical Aspects of Ionic Liquids; John Wiley \& Sons, Inc.: Hoboken, NJ, 2005.

(9) Ozawa, R.; Hamaguchi, H. Chem. Lett. 2001, 30, 736.

(10) Muldoon, M. J.; McLean, A. J.; Gordon, C. M.; Dunkin, I. R. Chem. Commun. 2001, 2364.

(11) Gordon, C. M.; McLean, A. J. Chem. Commun. 2000, 1395.

(12) Álvaro, M.; Ferrer, B.; Gracía, H.; Narayana, M. Chem. Phys. Lett. 2002, 362, 435.

(13) Wakasa, M. J. Phys. Chem. B 2007, 111, 9434.

(14) Hamasaki, A.; Nishizawa, K.; Sakaguchi, Y.; Okada, T.; Kido, G.; Wakasa, M. Chem. Lett. 2005, 34, 1692.

(15) Hamasaki, A.; Sakaguchi, Y.; Nishizawa, K.; Kido, G.; Wakasa, M. Mol. Phys. 2006, 104, 1765.

(16) Wakasa, M.; Hayashi, H. J. Phys. Chem. 1996, 100, 15640.

(17) Sakaguchi, Y.; Hayashi, H.; Nagakura, S. J. Phys. Chem. 1982, $86,3177$.

(18) Wakasa, M.; Nishizawa, K.; Abe, H.; Kido, G.; Hayashi, H. J. Am. Chem. Soc. 1999, 121, 9191.

(19) Feed, J. H. Chemically Induced Magnetic Polarization; Muus, L., Atkins, P. W., McLauchlan, K. A., Pedersen, J. B., Eds.; D. Reidel: Dordrecht, The Netherlands, 1977; Chapter 19.

(20) Pedersen, J. B. J. Chem. Phys. 1977, 67, 4097.

(21) Hamaguchi, H.; Ozawa, R. Adv. Chem. Phys. 2005, 131, 85

(22) Wang, Y.; Voth, G. A. J. Phys. Chem. B 2006, 110, 18601.

(23) Nishikawa, K.; Wang, S.; Katayanagi, H.; Hayashi, S.; Hamaguchi, H.; Koga, Y.; Tozaki, K. J. Phys. Chem. B 2007, 111, 4894.

(24) Funston, A. F.; Fadeeva, A. F.; Wishart, J. F.; Castner, E. W., Jr. J. Phys. Chem. B 2007, 111, 4963.

(25) Hayashi, H.; Nagakura, S. Bull. Chem. Soc. Jpn. 1984, 57, 322.

(26) Nakamura, Y.; Igarashi, M.; Sakaguchi, Y.; Hayashi, H. Chem. Phys. Lett. 1994, 217, 387.

(27) Nishizawa, K.; Sakaguchi, Y.; Abe, H.; Kido, G.; Hayashi, H. Mol. Phys. 2002, 100, 1137.

(28) Wakasa, M.; Hayashi, H.; Mikami, Y.; Takada, T. J. Phys. Chem. 1995, 99, 13181. 